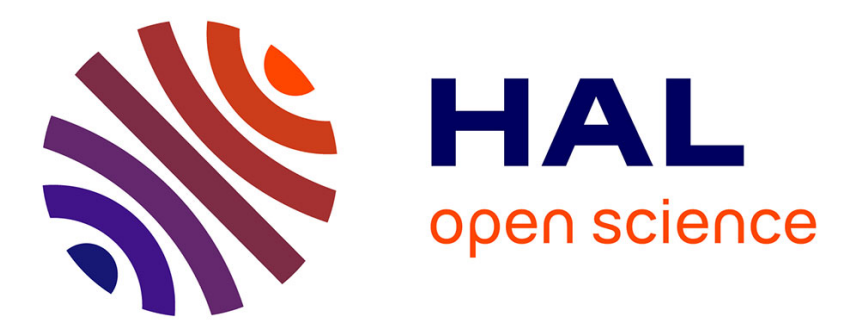

\title{
Measurement of the Interactions between Macroscopic Surfaces Inducing a First-Order Phase Transitions: the Sponge-Lamellar Transformation
}

\author{
D. Antelmi, P. Kékicheff, P. Richetti
}

\section{- To cite this version:}

D. Antelmi, P. Kékicheff, P. Richetti. Measurement of the Interactions between Macroscopic Surfaces Inducing a First-Order Phase Transitions: the Sponge-Lamellar Transformation. Journal de Physique II, 1995, 5 (1), pp.103-112. 10.1051/jp2:1995116 . jpa-00248133

\section{HAL Id: jpa-00248133 https://hal.science/jpa-00248133}

Submitted on 1 Jan 1995

HAL is a multi-disciplinary open access archive for the deposit and dissemination of scientific research documents, whether they are published or not. The documents may come from teaching and research institutions in France or abroad, or from public or private research centers.
L'archive ouverte pluridisciplinaire HAL, est destinée au dépôt et à la diffusion de documents scientifiques de niveau recherche, publiés ou non, émanant des établissements d'enseignement et de recherche français ou étrangers, des laboratoires publics ou privés. 
Classification

Physics Abstracts

$6470 \mathrm{M}-68.15-68.45 \mathrm{D}$

\title{
Measurement of the Interactions between Macroscopic Surfaces Inducing a First-Order Phase Transition: the Sponge-Lamellar Transformation
}

\author{
D. A. Antelmi $\left({ }^{1}\right)$, P. Kékicheff $\left({ }^{1}\right)$ and P. Richetti $\left({ }^{2}\right)$ \\ ( $\left.{ }^{1}\right)$ Department of Applied Mathematics, Research School of Physical Sciences and Engineering, \\ Australian National Unıversity, Canberra, A.C.T. 0200, Australia \\ ( $\left.{ }^{2}\right)$ C R P.P - C.N R.S., Château Brivazac, Avenue Schweitzer, 33600 Pessac, France
}

(Received 12 September 1994, accepted in final form 2 November 1994)

\begin{abstract}
The interactions between two macroscopic walls immersed in an isotropic symmetric sponge phase $\left(L_{3}\right)$ at different volume fractions, $\Phi$, were studied with a surface force apparatus At temperatures well below the lamellar $\left(\mathrm{L}_{\alpha}\right) / \mathrm{L}_{3}$ bulk transition, the force-distance profile is weakly oscillatory with a period that is twice the correlation length $(\xi)$ of the $\mathrm{L}_{3}$ phase measured from SAXS. The oscillations are superimposed on an exponentially attractive background with an order correlation length of 2-4 cell sizes of the sponge structure. When the temperature is raised, a first-order phase transition to a lamellar structure can be induced for separations below a threshold. Another oscillatory interaction results which has a period that is twice the reticular distance for an $\mathrm{L}_{\alpha}$ phase of similar $\Phi$. In addition, the thickness of the induced lamellar film increases (reversibly) with temperature. The compressibility modulus extracted from the oscillatory interaction is consistent with that expected for lamellae stabilized by undulation forces.
\end{abstract}

Recently both experimental and theoretical effort has been devoted to the physics of confined fluids. The phase equilibria of a confined fluid may be richer than that of a bulk fluid given that the free energy receives additional contributions [1]. For a confined fluid at a given temperature, the separation between the walls, $D$, can be used as the tuning variable to drive a phase transition in the same way as the temperature is used in bulk for the same fluid. For walls interacting with the fluid only vra an ordering potential, pretransitional effects can be induced under confinement on approaching the corresponding ordered phase domain [2-6]. When the bulk phase transition is second- order, the thickness of the pre-ordered film wetting the walls grows continuously and diverges at the bulk transition [3] (critical wetting [7]). On the other hand when it is first- order, the pretransitional regime is abruptly lost at a threshold separation, $D^{*}$. The preordered fluid transits discontinuously over its whole thickness towards the ordered state (first-order wetting [7]) whereas the bulk phase remains in the original state [8]. When the walls are preferentially wetted by the high-temperature disordered phase, the transition in the confined medium is then delayed [9]. 
Experimentally, there has been a great deal of work concerning thın films in the vicinity of both second- and first-order phase transitions in flat geometries (ordering of the film) [3]. More recently an extension to porous media has been carried out [10]. In this situation there is an interplay between the ordering of the complex fluid (liquid crystals for example) via its interactions with the substrate, and a tendency towards disorder $v a$ the induced defects needed to fill out the tortuous topology.

A less explored issue is the pretransitional effect on the interactions between the confining walls. Consider the case where two similar walls that impose symmetric boundary conditions interact with a fluid via a contact potential (zero range). The macroscopic surfaces are attracted to each other, since the thinner the confined film becomes the closer the film approaches a uniform state which is the thermodynamic steady state. The range of the attraction is related to the correlation length of the order parameter of the induced phase. The theory was first addressed by Marčelja and Radić [11] for a second-order transition and recently extended to a smectic transition by de Gennes [12]. First-order transitions have also been considered by Poniewierski and Sluckin [9]. To our knowledge, only a few studies have dealt with the measurement of the force between the walls near a phase transition. Horn et al. [13] measured interactions between mica surfaces confining a droplet thermotropic liquid crystal. They discussed their results in terms of pretransitional effects although the study was carried out at fixed temperature. Such a study was provided recently in the case of a second-order phase transition in a lyotropic system [14]. The present work completes the latter study as it investigates the case of a first-order isotropic/smectic phase transition for fluid membranes comprised of self-assembled surfactant molecules.

Here the chosen system offers additional interest to theory as it is an experimentally realized system of fluctuating surfaces. In a dilute lamellar phase $\left(\mathrm{L}_{\alpha}\right)$ the bilayers are stacked with a smectic order, while in an isotropic "sponge" phase $\left(\mathrm{L}_{3}\right)$ they form a multiply connected dividing surface. The present work investigates the sodium bis(2- ethyl hexyl) sulfosuccinate (AOT) / brine system which has an $\mathrm{L}_{3}$ and an $\mathrm{L}_{\alpha}$ phase located adjacent to each other in the phase diagram $[15,16]$. Previous studies have shown that in both phases the AOT bilayer has a similar thickness, $\delta=1.9 \mathrm{~nm}[17]$. The salinity can be used as the tuning variable to induce the phase transition. Alternatively at fixed composition, one may go from $\mathrm{L}_{3}$ to $\mathrm{L}_{\alpha}$ by increasing the temperature $(T)$, so the temperature difference from the bulk lamellar transition, $\Delta T=T-T_{\mathrm{L}_{\alpha}}$, can be used as the control parameter.

By varying the separation between two macroscopic surfaces using a surface force apparatus (SFA) [18], not only can one measure the force-distance profile, but also the affect on the interaction when the walls favour one phase $\left(\mathrm{L}_{\alpha}\right.$ in this case) over the other. Insights on the structural characteristics of the two phases can be extracted from the force curve measured in each of the two regimes where only one phase exists. Note that a recent study [19] has dealt with the same experimental system, but the variation of temperature was not carried out and the influence of the phase transition could not be identified. Some gross experimental features reported agree with our own observations but several important differences are noted for the two oscillatory regimes. In particular, our observations show that contrary to the work of Petrov et al. [19], the oscillatory force profile at small separations (whose extent depends on temperature) has a periodicity depending on $\Phi$. Furthermore, at large separations, an attractive background is observed, which was not pointed out by Petrov et al. [19].

\section{Experimental}

One must be aware of various experimental difficulties and artefacts when force measurements on surfactant systems are carried out with the SFA. First, AOT is known to degrade with time 
and the location of phase boundaries may be shifted by the presence of impurities (especially important for phases with a narrow extent in concentration) [20]. To ensure reproducibility in the samples, AOT (Fluka AG purum) was purified following a procedure described previously [21]. It is also known that halide ions (in particular $\mathrm{Cl}^{-}$) at high concentrations lead to degradation of the silver layers that are evaporated onto the back of each mica sheet to form the optical cavity in the SFA [18]. Although $\mathrm{NaCl}$ has been the more common salt used in studies of the AOT system, to avoid degradation of the silver/substrate interface, $\mathrm{NaNO}_{3}(\mathrm{BDH}$ Anal R; dried in vacuo) was used in preference [22]. Samples were prepared simply by weighing each component into a flask which was sealed and allowed to equilibrate for several days with frequent shaking. Prior to injection into the SFA and at the completion of the experiment the sample was checked by optical microscopy under crossed polarisers and by SAXS. The bulk $\mathrm{L}_{3} / \mathrm{L}_{\alpha}$ transition temperature was determined within $\pm 0.5^{\circ} \mathrm{C}$ and this set the upper limit for the force runs so that the bulk phase inside the SFA was always in the sponge state.

An additional problem often encountered when working with aqueous solutions of anionic surfactants is the degradation of the mica/silver interface. This can be monitored by observing the occurrence of coloured interference bands in the mica under magnification. This effect could be seen within a few hours after injection of the AOT/brine system. So to avoid impairment of the experiment, silica surfaces were used instead of mica. Thin silica (Suprasil, Heraeus) sheets were prepared following the method of Horn et al. [23]. Immediately prior to installation into the SFA, the silica surfaces were treated with a water plasma (18 MHz, $10 \mathrm{~W}$ for $30 \mathrm{~s}$, $P_{\mathrm{H}_{2} \mathrm{O}}=0.065$ Torr, $P_{\mathrm{Ar}}=0.022$ Torr) to remove any organic contaminants. The procedure developed by Horn and Smith for the asymmetric interferometer [24] was used to calculate the surface separation. By using silica surfaces the problems of degradation were avoided and experiments could be carried out over days or weeks allowing time to investigate the effects of varying temperature (from $16^{\circ} \mathrm{C}$ up to $30^{\circ} \mathrm{C}$ ). The temperature in the enclosure surrounding the SFA was controlled to $\pm 0.03^{\circ} \mathrm{C}$ resulting in minimal thermal drift of the surfaces during runs that lasted several hours. Reproducibilty between runs was excellent and the effects of temperature changes were reversible.

Comparison of the contact position at equilibrium in surfactant solutions with that of bare silica indicates that each silica surface is coated with a water layer followed by an AOT bilayer. For example, at $\Phi=0.135$ analysis of the refractive index obtained from the fringes of equal chromatic order indicates that below $20 \mathrm{~nm}$ two AOT bilayers exist, each separated from the silica wall by a $4-5 \mathrm{~nm}$ water layer. This forms a hard wall that can only be disrupted under high applied loads.

\section{Results}

The force-distance profiles exhibit similar features over the range of membrane fraction ( $\Phi=$ $0.09-0.18$ ) studied. For all volume fractions, a change in temperature induces similar effects and trends. The overall results can be illustrated by the data at $\Phi=0.135$ (Figs. 1-3).

For any $\Phi$, at temperatures well below the bulk transition to the $\mathrm{L}_{\alpha}$ phase $\left(\Delta T \approx-8^{\circ} \mathrm{C}\right)$, spatially decaying oscillatory forces superimposed on a weak attractive background are observed down to the hard wall (see above). Three main features characterize the oscillations of this regime, hereafter called the " $\mathrm{L}_{3}$ regime" (Fig.1):

(i) the peak-to-peak magnitude is always weak $(<0.05 \mathrm{mN} / \mathrm{m})$ and decreases as the membrane fraction is diluted;

(ii) the periodicity (separation between adjacent minima or maxima), $d_{\mathrm{L}_{3}}$, remains quite constant at large separations and is equal to about twice the characteristic length, $\xi$ (as measured for bulk samples from SAXS): $d_{\mathrm{L}_{3}}=(2.0 \pm 0.2) \xi$, but may show a reduction at smaller 


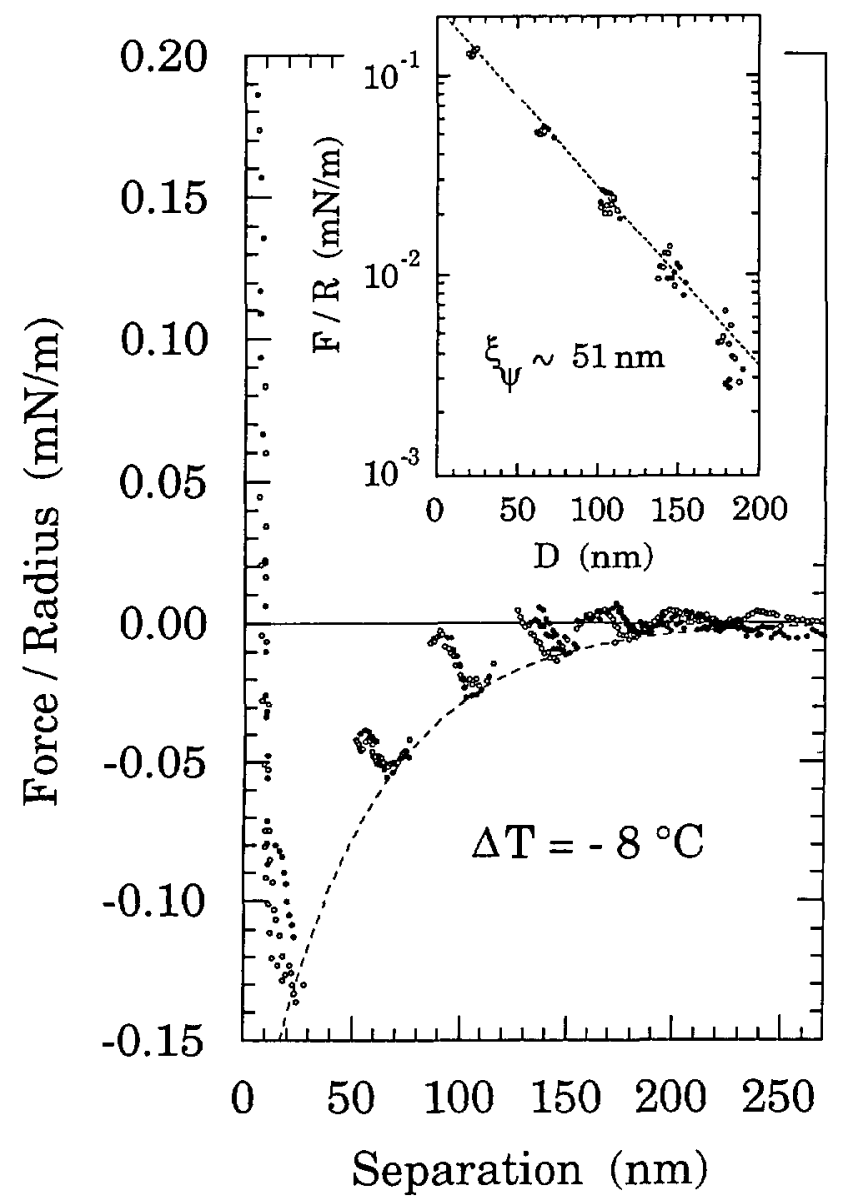

Fig. 1. - Forces $F$ (normalized by the mean radius of curvature $R$ of the surfaces) as a function of separation between two silica surfaces immersed in an AOT/brine sponge phase $(\Phi=0.135)$ at $T=20^{\circ} \mathrm{C}\left(\Delta T=-8{ }^{\circ} \mathrm{C}\right.$ below the $\mathrm{L}_{3} / \mathrm{L}_{\alpha}$ bulk transition temperature). The confined film remains in the $\mathrm{L}_{3}$ phase over the whole range of separation. The osclllations measured on compression $(\bullet)$ or on separation $(0)$ have a periodicity $d_{\mathrm{L}_{3}}=40 \pm 4 \mathrm{~nm} \approx 2 \xi(\xi$, characteristic length from SAXS). The munima are superimposed on an exponentially attractive background (dashed-line) with an order correlation length $\xi_{\Psi} \approx 2-3 \xi$ (inset).

separations (for the last oscillation). Note that for the weakest oscillations, the shape can be described continuously, as the gradient of the force remains smaller than the spring constant (stiffness $K$ ). However, when $\partial F / \partial D \geq K$, intrinsic unstable regimes occur [18] and the surfaces jump from one stable position to the next with a duration that can last up to a few minutes;

(iii) compression or dilation of the confined thin film between walls is not always fully reversible. Despite the fact that both the periodicity and the magnitudes of the oscillations are the same, independent of the direction of movement of the surfaces, the force-distance profiles obtained on approach or on separation can be shifted by a fraction of period.

When $\Delta T$ is reduced, a new regime appears for films thinner than a threshold separation, $D_{\mathrm{in}}^{*}$ (Fig. 2). The transition to this new regime is signalled by the occurrence of a large repulsive force on compression. However, for a given temperature, if the separation is never 


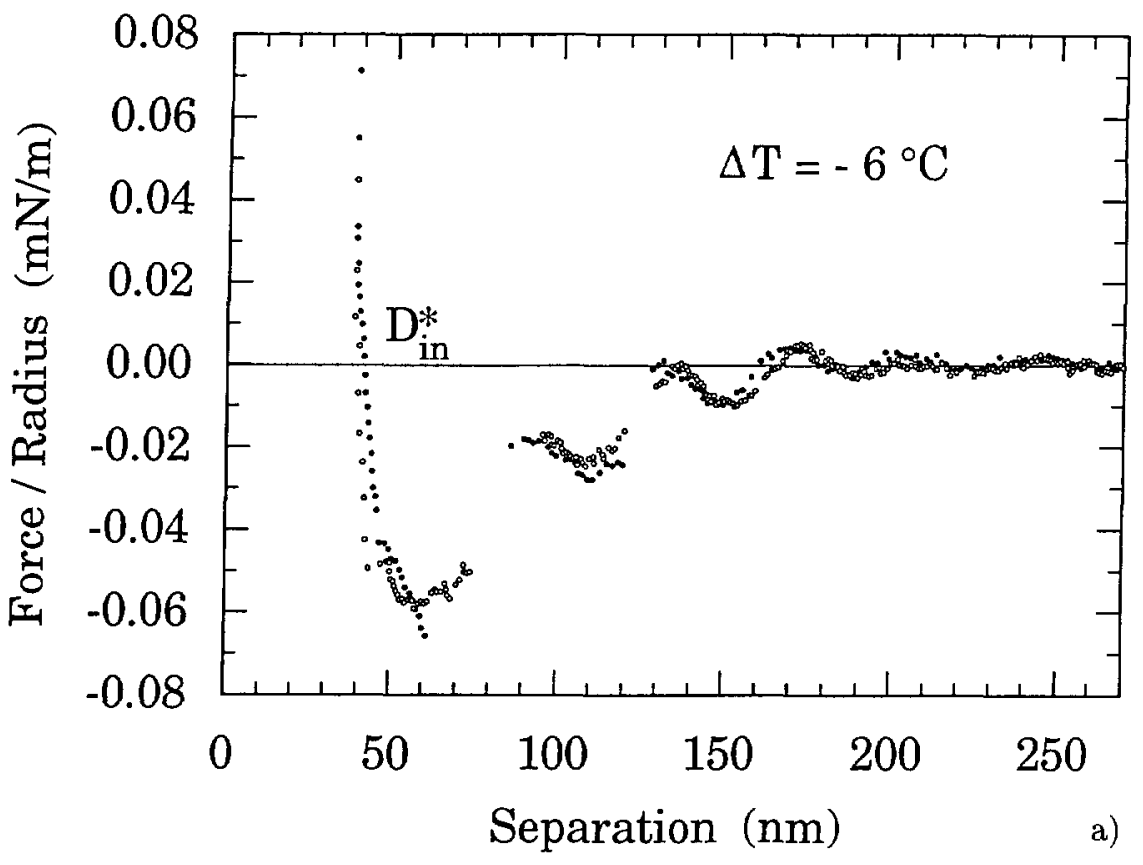

Fig 2. - a) $\Delta T=-6{ }^{\circ} \mathrm{C}\left(T=22{ }^{\circ} \mathrm{C}\right)$. Upon reducing the surface separation (o), the $\mathrm{L}_{3}$ regime is interrupted at $D_{1 \mathrm{n}}^{*}$ where the confined system transits to an $\mathbf{L}_{\alpha}$ structure. However, if the separation is not reduced below $D_{\mathrm{in}}^{*}$, the force profile for the outwards movement (o) shows that the confined fluid remains in the sponge state. Note that the $\mathrm{L}_{3}$ regıme is quite independent of $T$ (compare with Fig 1) b) $\Delta T=-4{ }^{\circ} \mathrm{C}\left(T=24{ }^{\circ} \mathrm{C}\right)$ : having reduced the separation (solid symbols: $\bullet \bullet$ ) below $D_{1 n}^{*}$, the induced $L_{\alpha}$ structure will transit back to the $L_{3}$ only if the surfaces are separated (empty symbols: $\square$, o) beyond $D_{\text {out }}^{*}$. The figure shows two experiments where the surfaces were separated after having described two $(n, \square)$ or only one $(\bullet, 0)$ of the $\mathrm{L}_{\alpha}$ oscillations. The periodicity measured between two consecutive minima of the osclllations indicates a Burgers vector $b=2$. Note the reversibilty of $D_{\mathrm{ln} / \text { out }}^{*}$, and the overall force profiles by comparing the curves obtained after increasing the temperature $(\square, \square)$ from $20^{\circ} \mathrm{C}$ (Fig 1) or after lowering $(\bullet, 0)$ from $26{ }^{\circ} \mathrm{C}$ (Fig 2c). In addition, the data for $(\square, \square)$ were measured using a different contact position on the silica to show the reproducibility between surface positions. c) $\Delta T=-2{ }^{\circ} \mathrm{C}\left(T=26{ }^{\circ} \mathrm{C}\right)$ : As the bulk transition is approached $\left(28^{\circ} \mathrm{C}\right)$, the $\mathrm{L}_{\alpha}$ film thickens at the expense of the $\mathrm{L}_{3}$ structure in the confined gap $\left(D_{\mathrm{in}}^{*} \approx 50,65,130 \mathrm{~nm}\right.$ at $\Delta T=-6,-4,-2{ }^{\circ} \mathrm{C}$ respectively). The return to the $\mathrm{L}_{3}$ regime is increasingly delayed as $|\Delta T|$ is reduced indicating the first-order nature of the phase transition $\left(D_{\text {out }}^{*} \approx D_{1 \mathrm{n}}^{*}\right.$ at $\Delta T=-6{ }^{\circ} \mathrm{C}$, but $D_{\text {in }}^{*}<D_{\text {out }}^{*} \approx 130,240 \mathrm{~nm}$ at $\Delta T=-4,-2{ }^{\circ} \mathrm{C}$ ). The inwards run is shown with solid symbols $(\bullet)$ and the outwards run is shown with empty symbols $(a)$.

reduced below the threshold $D_{\mathrm{in}}^{*}(T)$, the force profile exhibits the same features (i) to (iii) seen at the lowest temperature (Fig. 2a). Note that the oscillations have both their magnitude and periodicity, $d_{\mathrm{L}_{3}}$, independent of $T$. In other words the $\mathrm{L}_{3}$ regime is totally independent of the threshold $D_{1 \mathrm{n}}^{*}(T)$ provided that the separation between the surfaces has never decreased below $D_{\text {in }}^{*}$.

Below $D_{1 \mathrm{n}}^{*}(T)$, a different oscillatory behaviour is experienced with the minima of the oscillations located at an integral multiple of the reticular distance, $d$ (obtained from SAXS) for a bulk lamellar sample having a similar volume fraction. Hence this regime is referred to as the " $\mathrm{L}_{\alpha}$ regime". Remarkably, the distance between two consecutive minima is twice the reticular 

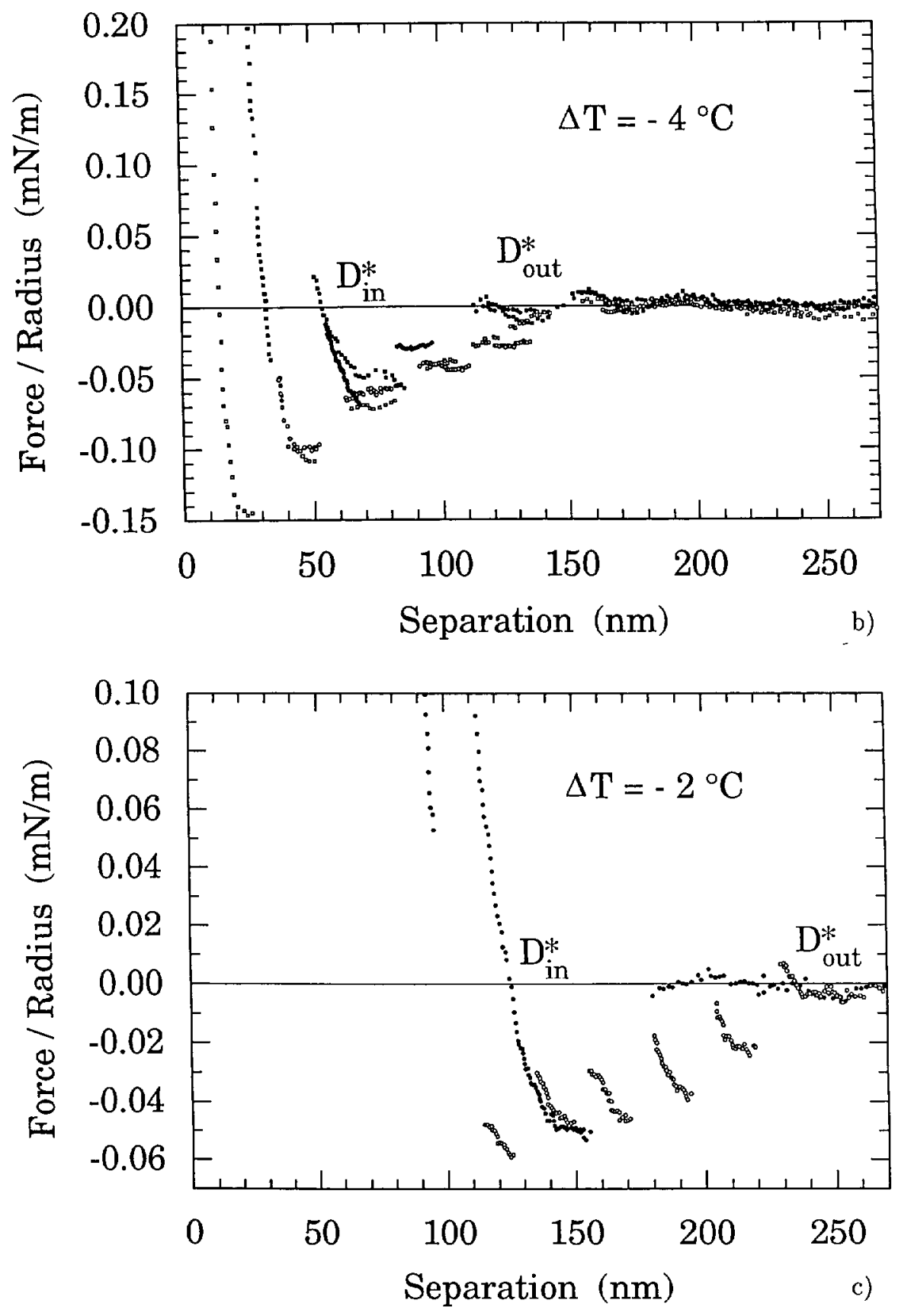

Fig. $2-($ Contınued $)$

distance: $d_{\mathrm{L}_{\alpha}}=b \cdot d$, with $b=2$. Note that for this surfactant system, no periodicity can be extracted from the measurement of the inward jump distances due to the non-equilibrium elastıc behaviour involved in the nucleation of edge dislocatıons upon compression (a complete discussion is given in [25]). The period, $d_{\mathrm{L}_{\alpha}}$, is therefore obtained from the minima of the 


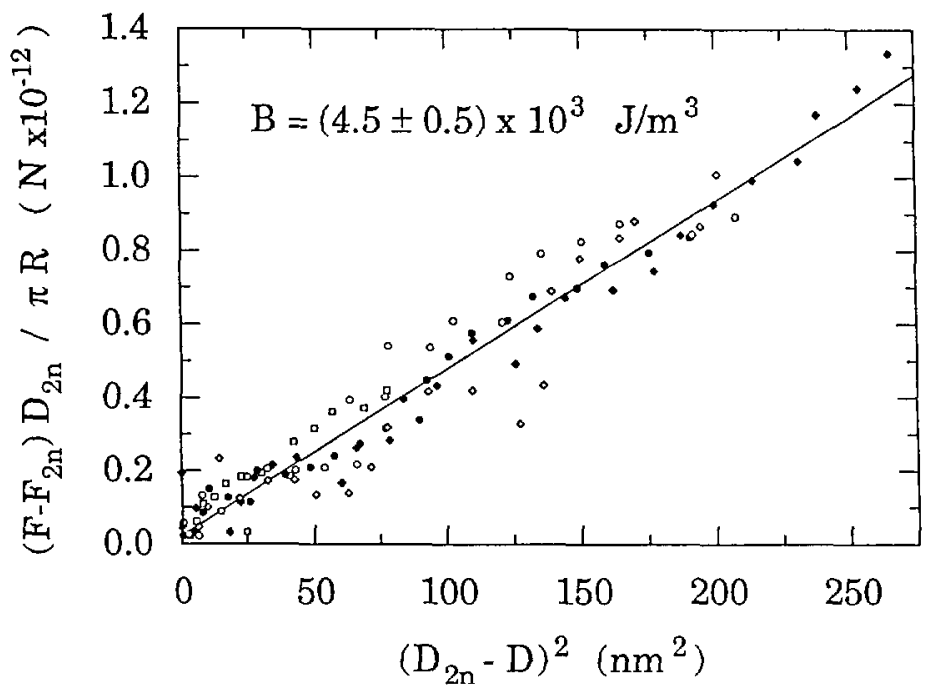

F1g. 3 - The compressibility modulus is extracted from the slope of $\left(F-F_{2 n}\right) D_{2 n} / \pi R v s .\left(D_{2 n}-D\right)^{2}$ using the Derjaguin approxımation where $F(D) / 2 \pi R$ is the elastic energy density of the $n^{\text {th }}$ parabola centered at $\left(D_{2 n}, F_{2 n} / 2 \pi R\right)$ (2n bilayers at zero stress confined in the gap). Data extracted from Figure 2c for the 5 th $(\square), 6$ th $(\bullet), 7$ th $(\circ), 8$ th $(\diamond)$, and 9 th $(\diamond)$ oscillations (from contact)

oscillations measured during an outwards force run (Figs. 2b-c).

Once initiated, the $\mathrm{L}_{\alpha}$ regime will disappear only when the surfaces are separated beyond $D_{\text {out }}^{*} \geq D_{\text {in }}^{*}$ (Figs. 2b-c). Remarkably, not only are both onsets, $D_{\text {in }}^{*}(T), D_{\text {out }}^{*}(T)$, a monotonic increasing function of temperature (and are reversible), but the hysteresis range $D_{\text {out }}^{*}(T)-$ $D_{\mathrm{n}}^{*}(T)$ also increases with temperature. Because the oscillations appear at locations virtually independent of $T$, their increasing number with $T$ is the signature of a thickening film with $\mathrm{L}_{\alpha}$ structure.

All the above features suggest a first-order phase transition induced by the walls where the $\mathrm{L}_{\alpha}$ structure is the preferred wetting phase. The first-order nature is confirmed by the lag in the transformation back to the $\mathrm{L}_{3}$ isotropic phase upon separating the surfaces (Figs. 2b-c).

\section{Analysis}

The discussion of the data falls into three parts: the $\mathrm{L}_{3}$ regime, the $\mathrm{L}_{3} / \mathrm{L}_{\alpha}$ phase transition, and the $\mathrm{L}_{\alpha}$ regime induced by the walls.

As its name indicates the sponge phase may be seen as a porous medium built of multiconnected bilayers having a uniform spatial distribution without any positional or orientational ordering. The sponge phase has a characteristıc length scale (pore size), $\xi$, which depends on the membrane volume fraction as $\xi \sim \delta / \Phi$. The preceding description has lead to the suggestion that the sponge phase can be seen as a melted cublc bilayer structure [26]. However, to interpret the scattering pattern, a more powerful description of the sponge phase has been proposed [27]. A continuous bilayer film partitions the solvent into two disjoint regions, referred to arbitrarily as "inside" (I) and "outside" (O). The difference in volume fractions of these regions is associated with an order parameter $(\Psi)$ of Ising-like symmetry. In a symmetric sponge phase, $\Psi=0$ whereas in an asymmetric sponge, $\Psi \neq 0$. As far as we know from existing systems, the transition between a lamellar phase and a symmetric sponge phase is the 
usual behaviour. In addition to the symmetric nature of the system used in this study, sponge phases are only found in the vicinity of swollen lamellar phases stabilized by undulation forces [28].

Now consider the results, it can be assumed that the silica surfaces induce locally, a layered order or prelamellar film adjacent to each wall comprised of thin slabs of alternate I and $\mathrm{O}$ solvent cells. Because a non-zero amplitude modulation is associated with this order, the wavenumber of the order parameter $\Psi$ far from the walls must be $q_{0} \approx 2 \pi / 2 \xi \propto \Phi$. In the vicinity of the walls, however, longer wavenumbers are expected for the first presmectic layers due to the nature of the membrane interaction, as hard walls have a screening effect on the undulation interaction $[29,30]$. Symmetric boundary conditions impose an exponentially decaying order parameter $\Psi$ (decay-length, $\xi_{\Psi}$ ), from the ordering walls down to the midplane. According to Marčelja and Radić [11], the order parameter distribution leads to an attraction with the same decay-length, the so-called order parameter correlation length. Figure 1 shows that the weak oscillations of the $\mathrm{L}_{3}$ regime superimpose on such an attraction. The typical decay-length $\xi_{\Psi}$ found is of the order of two to four times the characteristic length $\xi$ of the sponge phase $\left(\xi_{\Psi} \approx 51 \mathrm{~nm}\right.$ at $\Phi=0.135$; Fig. 1 inset). This is comparable to the value for correlations between membranes measured by light scattering for systems far away from the symmetric-asymmetric transition [31]. Now, the oscillatory behaviour can be understood as a packing effect induced under confinement when the separation between the walls is not an integer multiple of $\xi$, that is, elementary cells or presmectic layers confined in the gap. The periodicity $\approx 2 \xi$ indicates that the system responds by moving a pair of basic units of the $\mathrm{L}_{3}$ structure. No microscopic model (the $\mathrm{L}_{3}$ structure being three-dimensional) is at our disposal to explain such a behaviour, but the symmetric nature $(\Psi=0)$ of the sponge phase can be invoked. The periodicity of $2 \xi$, in order to protect the symmetry, would also be expected for an equivalent symmetric bicontınuous microemulsion comprised of oil and water in identical proportions

Phase transitions induced by walls are processes that are now well established both theoretically [8] and experimentally [2-6]. Here, the dramatic change in the force- distance profile for well defined threshold separations, $D^{*}(T)$, suggests such a transition between the $\mathrm{L}_{3}$ and the $\mathrm{L}_{\alpha}$ phases where the walls favour the $\mathrm{L}_{\alpha}$ over the $\mathrm{L}_{3}$. The hysteresis between $D_{\text {out }}^{*}(T)$ and $D_{\text {in }}^{*}(T)$ is evidence for a first-order phase transition, and arises from the finite area of the walls [1]. The effect is likely to be enhanced by both the peculiar topology of the walls (crossed-cylinders) in addition to the smectic nature of the confined structures (edge dislocations are induced). To first approximation, a linear attractive potential is expected between two parallel plates as soon as the phase transition is triggered off [25]. When the new phase is condensed between the plates, with a uniform distribution of the order parameter, the gradient terms vanish in a classical Landau-Ginzburg free energy. Unfortunately correspondence with the experimental crossed-cylinder geometry is not straightforward. Indeed, the usual Derjaguin approximation [32] is not expected to hold since the attraction is long-range Although, our data do not allow us to speculate on the precise shape of this interaction between the walls, the attractive nature of the $\mathrm{L}_{\alpha}$ regime is well-established on the runs obtained when the walls are moved apart (Fig. 2). A more difficult contribution to evaluate is the effect of the confinement topology as the crossed-cylinder geometry imposes a varying separation. The $\mathrm{L}_{\alpha}$ phase is induced only in a central droplet surrounded by a $\mathrm{L}_{3}$ medium. Film tension effects may then be expected to contribute to the net interaction. Finally, the one-dimensional order of the lamellar structure would also contribute to the net interaction [25].

The elastic response of the stack of layered membranes to any applied strain resulting from surface separations different from an integral multiple of layer thickness gives rise to an oscillatory interaction [33]. Because of the crossed-cylinder configuration the lamellae have to contour 
the slightly curved surfaces and a network of edge dislocations is formed. The measured periodicity of the oscillations indicates that the edge dislocations have a Burgers vector $b=2$. The topology of this defect (handle joining two neighbouring bilayers) is not surprising as it arises naturally from the bicontinuous structure of the sponge phase. The compressibility modulus, $B$, can be extracted from the parabolic shape of the oscillations [33]. The analysis carried out at any temperature within the studied range (about $10^{\circ} \mathrm{C}$ ) gives almost the same result. Because of the attractıve background contribution, the fit is applied to the difference $\left(F-F_{2 n}\right) / R$, where $F_{2 n}$ is the non-zero force at the minima $D_{2 n}$ of the $n^{\text {th }}$ oscillation ( $2 n$ bilayers confined in the gap). At large separations, $B$ is not dependent on $n$ and the experimental data follow $D_{2 n}\left(F-F_{2 n}\right) / \pi R=B \Delta D_{2 n}^{2}$ (Fig. 3 for $n=5-9$ ). Data from each oscillation lie along a straight line of slope $B=(4.5 \pm 0.5) \times 10^{3} \mathrm{~J} / \mathrm{m}^{3}$ at $\Phi=0.135$ for $n>5$ (Fig. 3). Note that in the above relation, $b$ does not appear explicitly but affects the locations of the intersection of two adjacent parabolae: the larger $b$, the wider the parabolae are. The obtained estimate for $B$ is comparable with that measured from high-resolution X-ray scattering in other similar systems [34] A direct comparison with theory is also possible. For AOT in brine, the lamellae have been shown to be stabilized by undulation forces and $B=9 \pi^{2} /(64 \kappa)\left(k_{\mathrm{B}} T\right)^{2} d /(d-\delta)^{4}$ [28]. Assuming $\kappa$ of the order $k_{\mathrm{B}} T$, the Helfrich formula gives $B=5.7 \times 10^{3} \mathrm{~J} / \mathrm{m}^{3}$ at $\Phi=0.135$ in agreement with the measured value [35]. Furthermore, our data confirm a decrease of $B$ as $1 / d^{3}$ when the lamellar phase is swollen; $B=14.4,4.5,1.38, \times 10^{3} \mathrm{~J} / \mathrm{m}^{3}$, at $\Phi=0.183,0.135$, and $0.091 \mathrm{AOT}$ respectively, where the reticular distance is $9.5,12.5,20.0 \mathrm{~nm}$.

\section{Acknowledgments}

It is a pleasure to thank Roger Horn for his advice concerning the preparation of the silica surfaces and also for his help in the analysis of the interferometry. Thanks are also extended to Tim Sawkins for technical assistance and to Didier Roux for stimulating discussions.

\section{References}

[1] Evans R. and Marinı Bettolo Marconı U., J Chem. Phys. 86 (1987) 7138.

[2] Miyano K, Phys. Rev. Lett. 43 (1979) 51

[3] Als-Nielsen J, Christensen F., Pershan P. S , Phys. Rev Lett. 48 (1982) 1107

[4] Ocko B. M, Braslau A., Pershan P S, Als-Nielsen J, Deutsch M., Phys. Rev. Lett. 57 (1986) 94

[5] Iannacchıone G. S., Finotello D., Phys. Rev. Lett. 69 (1992) 2094.

[6] Shı Y., Cull B , Kumar S, Phys. Rev. Lett. 71 (1993) 2773.

[7] Shick M., Lıquids at interfaces, Vol Sess. XLVIII, 1988, ch. 9, J Charvolın, J F Joanny and J. Zinn-Justin Eds (Elsevier Scı Publ., B.V., Les Houches, 1990).

[8] Sheng P., Phys Rev. Lett. 37 (1976) 1059; Phys. Rev. A 26 (1982) 1610.

[9] Ponewierski A., Sluckin T. J., Lıq Cryst. 2 (1987) 281.

[10] Vinches C., Coulon C., Roux D., J Phys. II France 2 (1992) 453.

[11] Marčelja S., Radić N., Chem. Phys, Lett. 42 (1976) 129.

[12] de Gennes P.-G., Langmuir 6 (1990) 1448.

[13] Horn R. G, Israelachvili J. N., Perez E, J. Phys. France 42 (1981) 39.

[14] Moreau L, Richetti P, Barois P, Phys. Rev. Lett. (December 1994) in press 
[15] Fontell K., Colloid dispersions and micellar behaviour; ACS Symposium Series (Amerıcan Chemical Society, Washington D.C., 1975) p. 270.

[16] Miller C. A., Ghosh O., Langmuir 2 (1986) 321.

[17] Strey R., Jahn W., Skouri M., Porte G., Marıgnan J., Olsson U., Structure and dynamics of strongly interacting colloids and supramolecular aggregates in solution, S.-H Chen et al Eds. (Kluwer Acad Publishers (The Netherlands, 1992) pp. 351-363

[18] Israelachvili J. N, Adams G E., J. Chem. Soc Faraday Trans. 174 (1978) 975

[19] Petrov P., Olsson U., Christenson H K., Mıklavıc S , Wennerström H., Langmuır 10 (1994) 988.

[20] Kunieda H., Shinoda K., J. Collozd Interface Sci. 70 (1979) 577

[21] Parker J. L, Richettı P, Kékıcheff P., Sarman S, Phys. Rev Lett. 68 (1992) 1955.

[22] Kékicheff P., Marčelja S., Senden T. J., Shubin V. E, J. Chem Phys. 99 (1993) 6098.

[23] Horn R. G., Smith D. T., Haller W., Chem. Phys. Lett. 162 (1989) 404

[24] Horn R G, Smith D. T., Appl. Opt. 30 (1991) 59.

[25] Richetti P, Kékıcheff P., Baross P., J Phys France, submitted

[26] Balinov B , Olsson U, Soderman O., J. Phys. Chem. 95 (1991) 5931

[27] Cates M. E, Roux D., Andelman D., Milner S T., Safran S A., Europhys. Lett. 5 (1988) 733;

Cates M E, Physica A 176 (1991) 187

[28] Helfrich W, Z Naturforsch 33a (1978) 305

[29] Janke W., Kleinert H., Meinhart M , Phys Lett. B 217 (1989) 525.

[30] Kékicheff P., Christenson H K., Phys. Rev Lett 63 (1989) 2823.

[31] Coulon C., Roux D , Bellocq A.-M., Phys. Rev Lett. 66 (1991) 1709.

[32] Derjaguin B. V, Kollord Z 69 (1934) 155.

[33] Richetti P., Kékicheff P., Parker J. L., Ninham B. W., Nature (London) 346 (1990) 252.

[34] Roux D., Safinya C R., J. Phys France 49 (1988) 307

[35] However, taking $\kappa=3 k_{\mathrm{B}} T$ according to Skouri el al (Skouri M , Marignan J., Appell J., Porte G., J. Phys II France 1 (1991) 1121), the measured value for the compressibslity modulus is larger than that predicted by the Helfrich formula 